\title{
El aprendizaje basado en problemas como estrategia didáctica en el diseño de prácticas de Laboratorio Clínico y Biomédico
}

\section{Problem-based learning as a didactic strategy in the design of clinical and biomedical laboratory practices}

\author{
Carlos Martínez ${ }^{1}$, Diego Salmerón ${ }^{2}$, Nicanor Morales-Delgado ${ }^{3}$ y \\ Antonia Alonso ${ }^{1, *}$ \\ 1 Departamento de Anatomía Humana y Psicobiología. Facultad de Medicina. Universidad de \\ Murcia (Murcia); carlos.m.l@um.es. ORCID ID: https://orcid.org/0000-0002-4267-5175. \\ 2 Departamento de Ciencias Sociosanitarias, Universidad de Murcia, Murcia. CIBER \\ Epidemiología y Salud Pública (CIBERESP), Murcia. IMIB-Arrixaca, Murcia; dsm@um.es. \\ ORCID ID: https://orcid.org/0000-0002-4574-6880 \\ 3 Departamento de Histología y Anatomía. Facultad de Medicina. Universidad Miguel \\ Hernández (Alicante) nmorales@umh.es. ORCID: https://orcid.org/0000-0002-6716-9041 \\ 1 Departamento de Anatomía Humana y Psicobiología. Facultad de Medicina. Universidad de \\ Murcia (Murcia); antoniaaf@um.es. ORCID: https://orcid.org/0000-0002-8214-8054. \\ * Correspondencia: antoniaaf@um.es
}

Recibido: 9/11/20; Aceptado: 27/12/20; Publicado: 28/12/20

Resumen: El aprendizaje basado en problemas (ABP) se ha posicionado en los últimos años como un referente de estrategias de aprendizaje activo en el ámbito educativo de las Ciencias de la Salud. Nos permite crear nuevos escenarios que se asemejen al futuro laboral de los estudiantes. Con este propósito, hemos aplicado la metodología ABP en el diseño y evaluación de prácticas de laboratorio enmarcadas en el Ciclo Formativo de Grado Superior en Laboratorio Clínico y Biomédico, para favorecer la adquisición de conocimientos y alcanzar un aprendizaje significativo. Este estudio incluyó una muestra de 20 participantes que se agruparon en pequeños grupos de trabajo de 3-4 alumnos. Los alumnos realizaron cuatro prácticas de laboratorio, alternando la metodología tradicional y la $\mathrm{ABP}$, resolviendo a su finalización un cuestionario para cada una de ellas, así como una encuesta de satisfacción sobre la metodología docente aplicada y el papel del profesor. Las respuestas recogidas con estos cuestionarios permitieron comparar la eficacia de ambas metodologías en la mejora de los resultados de los alumnos. Nuestros datos indican una mejoría en los resultados de los cuestionarios finales de todas las prácticas, obteniéndose en el peor de los casos una media de las notas 0.98 puntos mayor con la metodología ABP. No obstante, debido al bajo tamaño muestral, los resultados solo fueron estadísticamente significativos en una de las prácticas. Por otra parte, el 95\% del alumnado consideró la metodología aplicada como motivadora. Por tanto, podemos concluir que la metodología ABP se sitúa como una alternativa adecuada y motivadora en la enseñanza de las Ciencias Biomédicas, dado que permite crear un ambiente colaborativo y acercar a los estudiantes a su futuro ámbito laboral.

Palabras clave: Aprendizaje Basado en Problemas; constructivismo; aprendizaje significativo; aprendizaje colaborativo; educación biosanitaria

Abstract: In recent years, problem-based learning (PBL) has become a reference for active teaching strategies in the field of Health Sciences Education. It allows us to 
create new scenarios that closely resemble the future working environment of students. To this end, we have applied the PBL methodology in the design and evaluation of laboratory practices framed in the Higher Degree Training Cycle in Clinical and Biomedical Laboratory to promote knowledge and achieve meaningful learning. This study included 20 students, which were divided into small workgroups (3-4 people each). They carried out four laboratory practices, alternating the traditional methodology and PBL, and answered an individual practice questionnaire and a survey on the applied teaching methodology and the role of the teacher. The responses collected were used to compare the effectiveness of both methodologies by improving the results of the students. Our data indicate an improvement in the final questionnaires of all practices performed with the PBL approach, obtaining in the worst case an average score of 0.98 points higher than the traditional one. However, due to the low sample level, the results were only statistically significant in one of the practices. On the other hand, 95\% of students considered the methodology applied as motivating. On the other hand, 95\% of students considered the methodology applied as motivating. Overall, we can conclude that the PBL methodology is an adequate and motivating alternative in Biomedical Sciences teaching since it creates a collaborative environment and brings students closer to their future professional environment.

Keywords: Problem-based learning; constructivism; significant learning; collaborative learning; biosanitary education

\section{Introducción}

La sociedad del siglo XXI se encuentra sujeta a cambios continuos que son consecuencias de la revolución tecnológica y de la globalización. Ante este escenario, el mercado laboral necesita y exige profesionales versátiles que, además de poseer una formación sólida en conocimientos de su especialización, sean capaces de adaptarse a las situaciones adversas y el continuo cambio, es decir, profesionales resilientes. La clave en la formación de este perfil de profesionales se encuentra en la educación centrada en la profesionalización del alumnado. En España, el sistema educativo actual, y en especial, la Educación Superior, no responde a estas necesidades del mercado laboral, debido, en gran medida, al retraso con el que se implantan metodologías educativas innovadoras y a la inercia con la que la enseñanza se ancla en la metodología tradicional (1). Como consecuencia, para el estudiante y su futuro profesional, la Educación Superior se experimenta como una etapa educativa cuya gran diferencia respecto a los niveles educativos medios previos es el aumento considerable de los contenidos y de la carga de trabajo.

Una excepción a este problema lo encontramos en la educación en Ciencias de la Salud, que experimentó una revolución innovadora en los métodos de enseñanza-aprendizaje aplicados en los centros universitarios, durante el siglo XX, con la incorporación de la ciencia en los currículos. Esta revolución no llegó a la Formación Profesional (FP) de orientación Biosanitaria, que actualmente presenta planes de estudio fragmentados y obsoletos, con enfoques técnicos limitados y automatizados, que no profundizan en la comprensión conceptual de los métodos y los protocolos utilizados (2). El nuevo marco educativo, promovido por el Espacio Europeo de Educación (EEES), busca superar estas deficiencias del sistema educativo a través del aprendizaje a lo largo de la vida y del "aprender a aprender". Ambos objetivos implican que se investigue y se apliquen nuevas metodologías 
de enseñanza-aprendizaje, así como que se innove en las aulas de FP (3). Se persigue que los docentes abandonen el método de enseñanza-aprendizaje memorístico, repetitivo y mecánico, en el que el alumnado tiene un papel pasivo, cuya única función es la de recibir información y relacionar conceptos por medio de situaciones estímulo-respuesta (4), para formar parte, de manera activa, de su propio proceso de enseñanza-aprendizaje, es decir, un aprendizaje constructivista $(3,5)$.

El aprendizaje constructivista pretende que el alumnado adquiera destrezas y conocimientos mediante el aprendizaje a través de la experiencia y la interacción con su entorno. Esto se consigue con actividades diseñadas para que vincule lo que aprende a situaciones de su vida cotidiana, elaborando e interpretando la información, lo que requiere indagar cuáles son los conocimientos previos del alumnado y realizar un diseño de las actividades adaptado a estos (5). El mayor referente del constructivismo es el aprendizaje significativo, que se basa en que el proceso de aprendizaje implica pensamiento y emociones, por lo que, cuando se considera a ambas en conjunto, el alumno comprende el significado de su experiencia, es decir, aprende. En este proceso también resultan importantes los docentes la estructura del currículo y el entramado social en el que se desarrolla el proceso educativo (6). El docente adapta las actividades de enseñanzaaprendizaje a los conocimientos previos de los alumnos, e incrementa su motivación y su autoestima $(7,8)$. Cuando el alumno hace uso de los conocimientos ya aprendidos para integrar los nuevos, tiene lugar una reconciliación integradora y una reorganización del conocimiento. Este proceso es necesario para poder discernir entre conceptos iguales y diferentes, y es así como el alumno construye su propio conocimiento (9).

El Aprendizaje Basado en Problemas (ABP), aplicada por primera vez en la resolución de casos clínicos por parte de estudiantes de Medicina (10), intenta aplicar el aprendizaje cooperativo y activo, a la resolución de problemas del mundo real en el proceso de enseñanza-aprendizaje de los alumnos (11). Se caracteriza porque centra el proceso de enseñanza-aprendizaje en el alumno, pudiéndose llevar a cabo con pequeños grupos de estudiantes, siempre bajo la supervisión de un docente, que queda en un segundo plano con un papel meramente orientativo. Además, los problemas planteados a los alumnos deben de estar relacionados con situaciones de la vida real, para ser utilizados como instrumentos para lograr los conocimientos y las aptitudes para resolverlos. Esto permite que el aprendizaje de la nueva información en los alumnos se produzca tras la realización del análisis y resolución de los problemas (12). El docente debe diseñar problemas que sean retos a resolver por los estudiantes, induciéndolos a comprometerse con su resolución, mediante la búsqueda, el análisis y la evaluación de la información (13-14). Debido a ello, es una metodología didáctica que responde a las necesidades formativas de la Formación Profesional. En este contexto, el diseño de prácticas de laboratorio con metodología ABP permite al docente motivar al alumnado y conseguir que comprenda en profundidad la finalidad de las prácticas que realizan. De este modo, y mediante el trabajo en grupos, los alumnos incorporaran los nuevos conocimientos a su estructura cognitiva previa, construyendo su propio conocimiento y colaborando entre ellos para alcanzar la resolución de los problemas (retos) propuestos por el docente. Es por ello que en el presente estudio nos propusimos como objetivo aplicar la metodología $\mathrm{ABP}$ en el diseño y evaluación de prácticas de laboratorio del módulo Análisis Bioquímico del Ciclo Formativo de Laboratorio Clínico y Biomédico como estrategia para favorecer la adquisición de conocimientos y 
alcanzar así un aprendizaje significativo. Hipotetizamos que la aplicación de $\mathrm{ABP}$ en este tipo de ciclos formativos es prácticamente inexistente y que el nivel de conocimiento adquirido con el método tradicional es más bajo.

\section{Métodos}

\subsection{Contexto socioeconómico del Centro Educativo}

Esta investigación se realizó durante el mes de febrero de 2020 como parte de las prácticas docentes del Máster en Formación del Profesorado, desarrollándose en el Instituto de Enseñanza Secundaria (IES) Miguel de Cervantes (Murcia). En este centro de enseñanza se imparten Educación Secundaria Obligatoria, Bachillerato y distintos Ciclos Formativos de Formación Profesional. Dispone de un edificio destinado exclusivamente a la FP y seis aulas taller del departamento de Sanidad, en el que se desarrollan las prácticas de los Ciclos Formativos de esta familia profesional.

\subsection{Características del Ciclo Formativo y de la Unidad de Trabajo}

Este trabajo se enmarca en el Ciclo Formativo de Grado Superior de Laboratorio Clínico y Biomédico, perteneciente a la familia profesional de Sanidad y se rige por el Real Decreto 771/2014, de 12 de septiembre, según el cual, posee el nivel 1 dentro del Marco Español de Cualificaciones para la Educación Superior. La obtención del título de Técnico Superior supone la cualificación profesional completa de Laboratorio de Análisis Clínicos (SAN124_3) y dos cualificaciones incompletas, la de Anatomía Patológica y Citología, proporcionando las Unidades de Competencia (UC) 0375_3 y UC0381_3 y la de Ensayos Microbiológicos y Biotecnológicos (QUI020_3), proporcionando la UC0055_3. Este Ciclo Formativo se divide en dos cursos, con un total de 6 módulos por curso, que se clasifican por la presencia o no de prácticas en el laboratorio (Tabla 1).

Tabla 1. Módulos del Ciclo Formativo de Laboratorio Clínico y Biomédico clasificados en función de las prácticas de laboratorio.

\begin{tabular}{|l|l|}
\hline \multicolumn{1}{|c|}{ Módulos con prácticas de laboratorio } & Módulos sin prácticas de laboratorio \\
\hline Técnicas generales de laboratorio & Gestión de muestras biológicas \\
\hline Biología molecular y citogenética & Fisiopatología general \\
\hline Análisis bioquímico & Formación y orientación laboral \\
\hline Técnicas de inmunodiagnóstico & Empresa e iniciativa emprendedora \\
\hline Microbiología clínica & Proyecto de laboratorio clínico y biomédico \\
\hline Técnicas de análisis hematológico & Formación en centros de trabajo \\
\hline
\end{tabular}

Nuestro estudio se desarrolló en el Módulo de Análisis Bioquímico que se imparte durante el segundo curso del Ciclo Formativo, y, concretamente, en la Unidad de Trabajo "Magnitudes: productos finales del metabolismo", que posee un importante carácter práctico.

\subsection{Participantes en el estudio}

La muestra del estudio estuvo formada por 20 estudiantes, de los cuales el $75 \%$ eran mujeres y el $25 \%$ restante hombres. La participación en las sesiones de clase fue inconstante, por lo que sólo participaron 15 alumnos (75\%) en todas las sesiones. Las edades de los alumnos estuvieron comprendidas entre los 19 y los 27 años. 


\subsection{Instrumentos de recogida de información}

Como instrumento de recogida de información se emplearon cuestionarios de preguntas. Se elaboró un cuestionario para cada práctica, siendo el número de preguntas de 6 en el caso de las prácticas para la determinación de creatinina y urea, 5 preguntas en el caso de la bilirrubina y 3 en el caso del ácido úrico. El tiempo para realizar la práctica y su complicación determinaron el número de preguntas por cuestionario. En todos los cuestionarios se elaboraron preguntas de dificultad similar, independientemente de su número y su diseño se relacionó con el fundamento, metodología, procedimiento y cálculos necesarios en el desarrollo de cada protocolo. El objetivo de estas preguntas fue comprobar si el alumnado comprendió cada una de las partes de las prácticas, por lo que se centraron en la totalidad de la práctica y no solo en la capacidad del alumno de seguir el protocolo. Además, los cuestionarios se basaron en los conocimientos transmitidos a los alumnos en la clase teórica previa a las prácticas. Los alumnos cumplimentaron los cuestionarios al finalizar todas las prácticas.

\subsection{Características del aula y materiales utilizados}

Las clases prácticas se realizaron en el laboratorio de Bioquímica y Hematología, que dispone de una superficie de $100 \mathrm{~m} 2$ y todo el equipamiento necesario para el correcto funcionamiento de las prácticas. Los puestos de trabajo se distribuyeron en cuatro banquetas dispuestas en filas de cuatro puestos de trabajo cada una, mientras que el material se organizó en armarios y cajones distribuidos por el laboratorio (Figura 1).

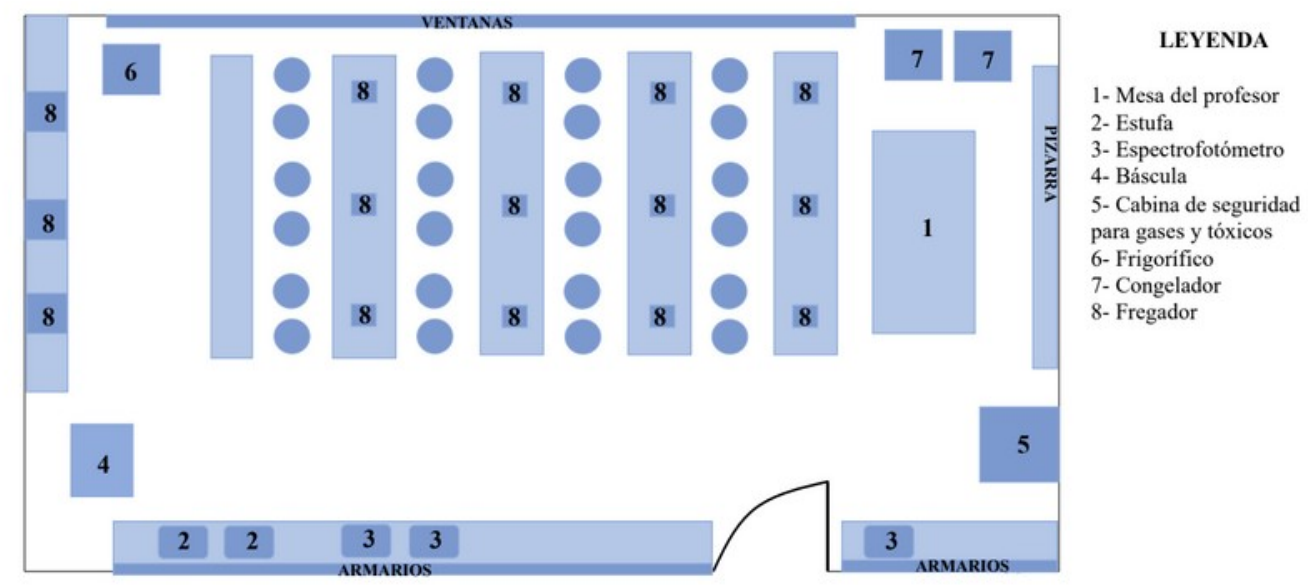

Figura 1. Plano del laboratorio de Bioquímica y Hematología del IES Miguel de Cervantes.

Todos los alumnos realizaron las prácticas con kits comerciales de análisis bioquímicos específicos para cada práctica, desarrollados por la casa Spinreacts (Girona, España). Cada kit contiene el protocolo de uso. Estos kits se utilizaron en las prácticas desarrolladas con ambas metodologías docentes: tradicional y ABP. Por el contrario, sólo en las prácticas en las que se aplicó metodología $\mathrm{ABP}$ los alumnos utilizaron material de apoyo, que consistió en el libro de texto de la editorial Altamar "Análisis Bioquímico" (ISBN 978-8416415-23-6), la presentación en formato PowerPoint utilizada por el docente en la explicación teórica la Unidad de Trabajo, y la aplicación informática para teléfonos móviles "Valores de laboratorio" (NoiaTech, Brasil). Esta aplicación es 
de uso intuitivo y sencillo. Proporciona información sobre los distintos valores de referencia de los diferentes analitos en hombres y en mujeres, así como un comentario de ellos en el caso de que se encuentre alto o bajo, permitiendo así conocer los valores de referencia de cada analito determinado en las prácticas (Figura 3).

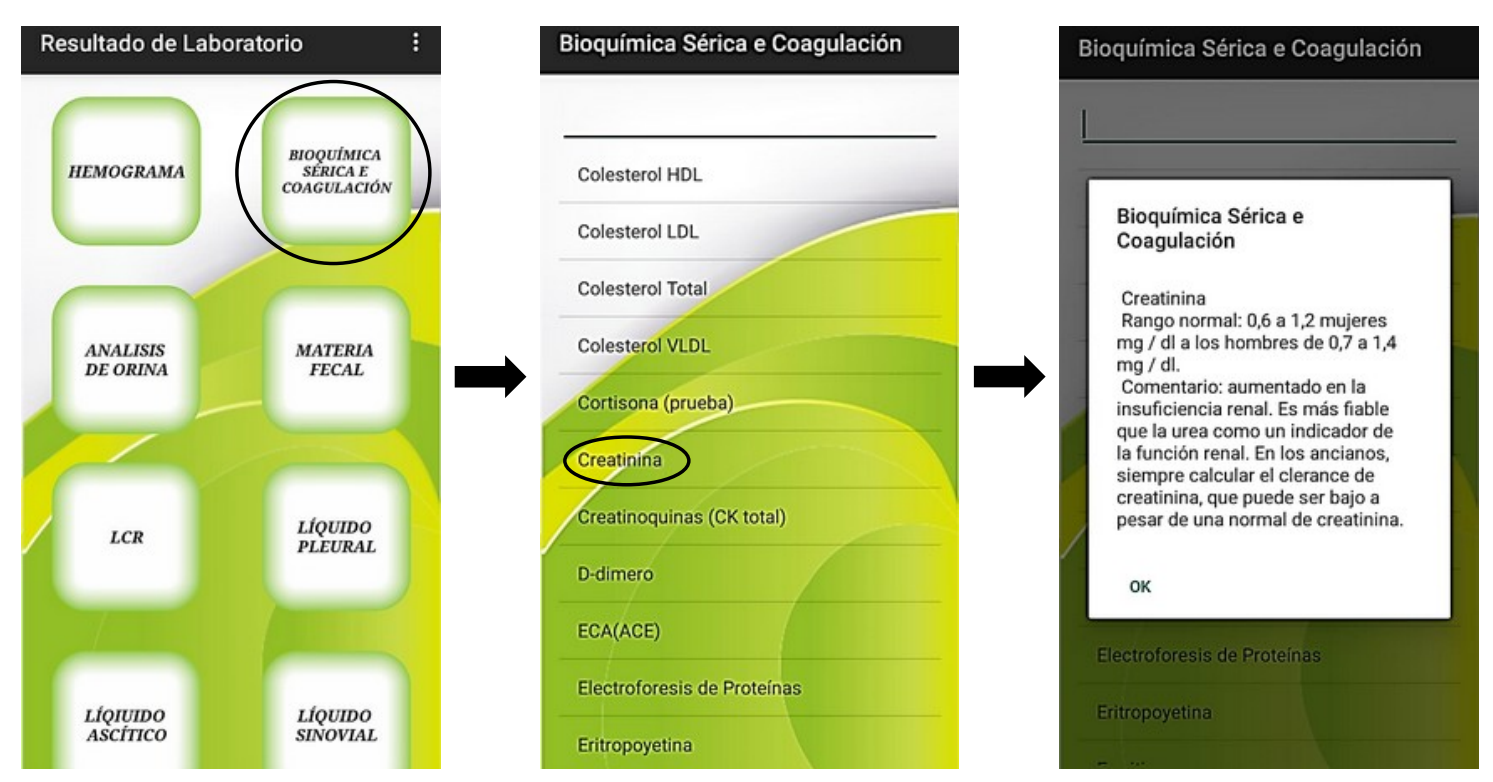

Figura 2. Imagen que recoge la interfaz y el funcionamiento de la aplicación móvil "Valores de Laboratorio" (Noia Tech).

\subsection{Diseño de la propuesta de intervención educativa}

La intervención educativa propuesta en este trabajo consistió en el diseño y aplicación de dos metodologías didácticas distintas en el desarrollo de prácticas de laboratorio sobre la determinación de productos finales del metabolismo, la considerada metodología "tradicional" y la metodología ABP. Los grupos de trabajo se establecieron al azar y estuvieron formados por 3-4 alumnos para promover el aprendizaje colaborativo entre ellos. A su vez, estos pequeños grupos se distribuyeron en dos grandes grupos, A y B. Todos los alumnos realizaron las prácticas tanto con metodología tradicional como con $\mathrm{ABP}$. Se decidió aleatoriamente qué prácticas iban realizar los integrantes de cada grupo (A o B) mediante una metodología didáctica "tradicional" o una metodología "ABP". Las prácticas de laboratorio escogidas para realizar este trabajo fueron la determinación de urea, ácido úrico, bilirrubina y creatinina, que forman parte de los contenidos de la Unidad de Trabajo "Productos finales del metabolismo", del Módulo de Análisis Bioquímico de este ciclo.

Las clases prácticas se impartieron en dos sesiones de 3 horas cada una. En cada sesión se desarrollaron, de forma simultánea, los protocolos de los cuatro kits utilizados (determinación de los valores de ácido úrico, creatinina, urea y bilirrubina). Los cuatro supuestos prácticos se agruparon en dos bloques: el Bloque 1, compuesto por las prácticas para la determinación de ácido úrico y creatinina, y el Bloque 2, constituido por las determinaciones de bilirrubina y urea. Durante la primera sesión se aplicó la metodología tradicional, resolviendo el profesor las dudas relacionadas con alguno de los pasos del protocolo o con el fundamento de la práctica. Durante esta sesión, el grupo A realizó las prácticas del Bloque 1, y el grupo B, las del Bloque 2. En la 
segunda sesión se intercambiaron los Bloques de prácticas y se aplicó la metodología $\mathrm{ABP}$, que consistió en la modificación de los protocolos dejando espacios vacíos en las explicaciones del procedimiento y en la inclusión de cuestiones basadas en las dudas planteadas durante la primera sesión. Los alumnos resolvieron las cuestiones y rellenaron los espacios vacíos trabajando en grupo, mediante un proceso de investigación en el que utilizaron el material de apoyo y la aplicación móvil disponible para la práctica. El docente sólo jugó un papel de apoyo, dando el visto bueno a las respuestas correctas e incentivando el pensamiento y la búsqueda de otras opciones mediante una pista cuando el alumnado no daba con la respuesta adecuada.

No todos los alumnos realizaron las cuatro prácticas. La Tabla 2 resume los alumnos que realizaron cada práctica y la metodología aplicada, en su caso. Al término de la segunda sesión, los alumnos de ambos grupos realizaron el cuestionario final sobre todas las prácticas.

\begin{tabular}{|c|c|c|c|c|c|c|c|c|}
\hline \multirow{4}{*}{\begin{tabular}{|c|} 
Grupo \\
Método \\
Alumnos \\
\end{tabular}} & \multicolumn{4}{|c|}{ Sesión 1} & \multicolumn{4}{|c|}{ Sesión 2} \\
\hline & \multicolumn{2}{|c|}{$\mathrm{A}$} & \multicolumn{2}{|c|}{ B } & \multicolumn{2}{|c|}{ A } & \multicolumn{2}{|c|}{ B } \\
\hline & \multicolumn{4}{|c|}{ Tradicional } & \multicolumn{4}{|c|}{ ABP } \\
\hline & \multicolumn{2}{|c|}{11} & \multicolumn{2}{|c|}{9} & \multicolumn{2}{|c|}{10} & \multicolumn{2}{|c|}{9} \\
\hline Práctica & $\mathrm{AU}$ & CR & $\mathrm{BR}$ & $\mathrm{U}$ & $\mathrm{BR}$ & $\mathrm{U}$ & $\mathrm{AU}$ & $\mathrm{CR}$ \\
\hline $\begin{array}{l}\text { Alumnos } \\
\text { presentes }\end{array}$ & 9 & 11 & 9 & 9 & 10 & 9 & 9 & 9 \\
\hline
\end{tabular}

Tabla 2. Distribución por sesiones de las prácticas y alumnos presentes en ellas. AU: ácido úrico; CR: creatinina; BR: bilirrubina; U: urea.

\subsection{Valoración de la respuesta educativa}

Para conocer la opinión del alumnado acerca de la intervención educativa, se elaboró una encuesta mediante la herramienta Formularios de Google (https://www.google.com/intl/es es/forms/about/). La encuesta se cumplimentó de manera anónima y constó de dos partes. La primera parte (Anexo I) consistió en una evaluación de la metodología ABP, con preguntas de respuesta cerrada validadas previamente $(16,17)$. La segunda parte constó de 5 preguntas de elaboración propia cuyo objetivo era recabar la opinión del alumnado con respecto a la acción docente. Las respuestas a estas preguntas eran del tipo escala Likert de 5 niveles ( $1=$ totalmente en desacuerdo hasta 5 = totalmente de acuerdo).

\subsection{Análisis estadístico de los resultados}

Los datos obtenidos con los cuestionarios finales se analizaron mediante tres procedimientos. En primer lugar, mediante un análisis descriptivo. En segundo lugar, se realizó un análisis de varianza a los resultados de los cuestionarios de cada una de las prácticas, para confirmar la igualdad de estas. Por último, se aplicó la prueba t-Student a las medias de las notas, para determinar el nivel de significación de los resultados. El test estadístico utilizado fue el test de la t-Student. Se contrastó la hipótesis de igualdad de varianza y en ninguna ocasión se pudo concluir la heterocedasticidad ( $p$-valor $>0.3$ ). No obstante, como los tamaños muestrales son pequeños, también se aplicó el test no paramétrico de Mann-Whitney para comparar las metodologías, y los resultados obtenidos fueron los mismos. Para realizar el análisis estadístico se utilizó el software IBM SPSS (SPSS Inc., V18.0, Chicago, USA). 


\section{Resultados} estudio.

En este apartado mostramos los resultados de nuestra población de

\subsection{Análisis descriptivo: metodología $A B P$ vs tradicional}

Para el análisis descriptivo de los resultados de los cuestionarios se calcularon la media de las calificaciones obtenidas, la desviación estándar y la nota mínima y máxima obtenida, para ambas metodologías docentes. Se observó que la mayoría de las notas máximas y de las notas mínimas obtenidas por los alumnos en cada práctica fueron más altas en aquellas en las que se aplicó la metodología $\mathrm{ABP}$, que en las que se impartieron con metodología tradicional. Los resultados se muestran en las Tablas 3.

\begin{tabular}{|c|c|c|c|c|c|c|c|c|}
\hline \multicolumn{4}{|c|}{ Metodología tradicional } & \multicolumn{3}{c|}{ Metodología ABP } \\
\hline & Media & D.E. & Mínimo & Máximo & Media & D.E. & Mínimo & Máximo \\
\hline AU & 6,3 & 2,02 & 3,3 & 10 & 8,9 & 1,65 & 6,7 & 10 \\
\hline BR & 5,08 & 2,20 & 2,0 & 8,0 & 6,6 & 2,11 & 4,0 & 10 \\
\hline CR & 5,45 & 2,58 & 1,7 & 8,3 & 6,67 & 2,88 & 1,7 & 10 \\
\hline U & 4,58 & 2,91 & 0 & 8,3 & 5,56 & 2,02 & 1,7 & 8,3 \\
\hline
\end{tabular}

Tabla 3. Valores de las variables con las metodologías tradicional y ABP. AU, ácido úrico; CR, creatinina; BR, bilirrubina; U, urea; n, número de alumnos; D.E., desviación estándar

La comparación de las medias de las notas obtenidas por los alumnos en los cuestionarios finales de las prácticas impartidas con metodología $\mathrm{ABP}$ vs. la metodología tradicional mostró una tendencia a la mejoría de las notas en las cuatro prácticas cuando se aplicó la metodología ABP. No obstante, estos resultados sólo fueron estadísticamente significativos en la práctica del ácido úrico, con una media de las notas de 2,6 puntos mayor para la metodología ABP, con $\mathrm{p}=0,009$ (Figura 3).

En los 3 supuestos prácticos restantes, las determinaciones de bilirrubina, creatinina y urea, también se observó una

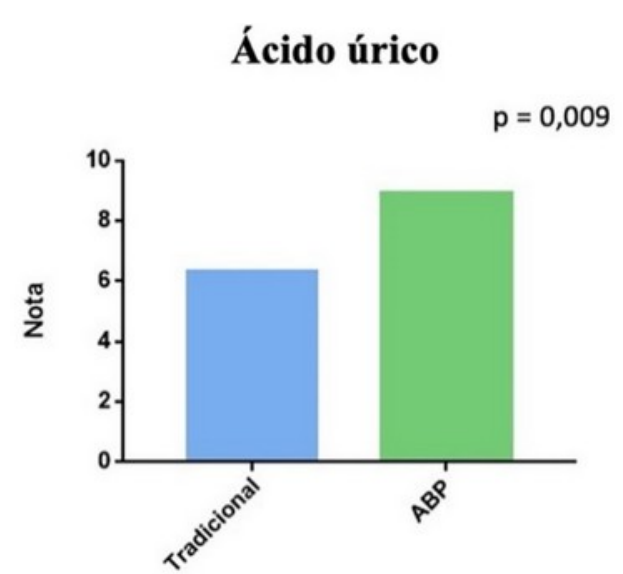

Figura 3. Comparación de las notas medias obtenidas mediante metodología tradicional y ABP en el cuestionario final de la práctica del ácido úrico. $\mathrm{p}=0,009$. mejoría de las notas con la metodología docente ABP frente a la tradicional. En la práctica de determinación de la bilirrubina se obtuvo una media de las notas de 1,52 puntos mayor con la metodología ABP que en la tradicional. En el caso de la creatinina, la media fue de 1,22 puntos mayor, mientras que sólo hubo una diferencia de 0,98 puntos en la práctica de la urea entre ambas metodologías. No obstante, como hemos mencionado previamente, estos resultados no fueron estadísticamente significativos, al ser los valores de $\mathrm{p}$ mayores de 0,05 (Figuras 4A-C). 

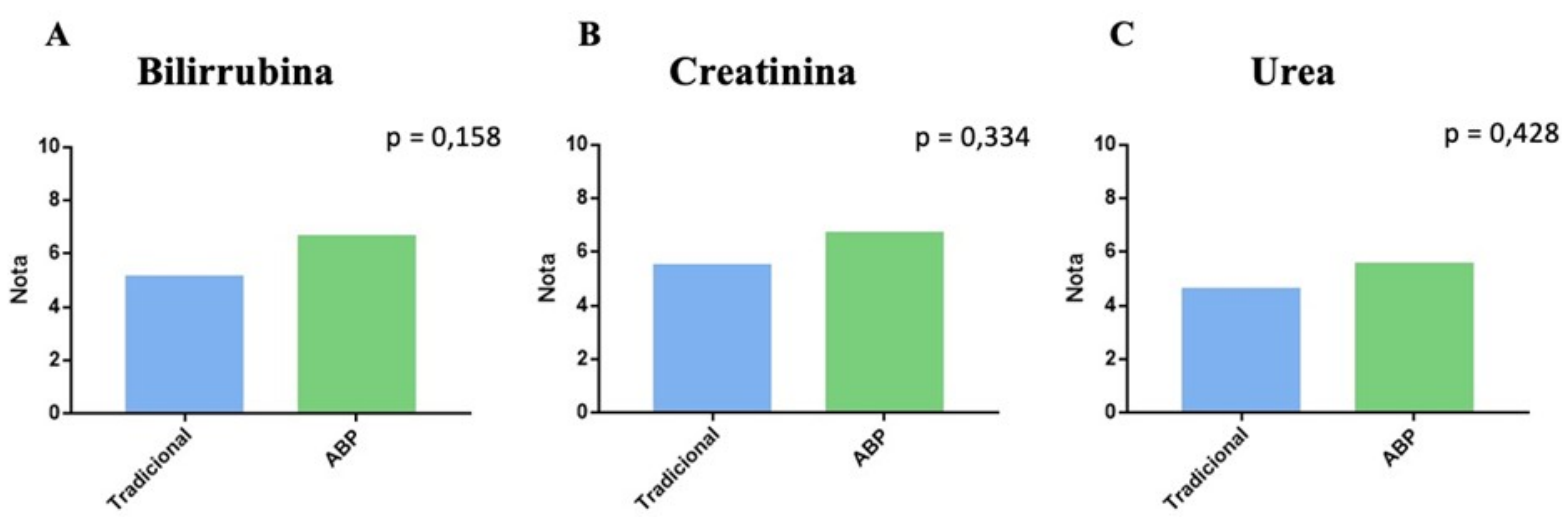

Figura 4. Comparación de las notas medias obtenidas mediante metodología tradicional y ABP en los cuestionarios finales de las prácticas. A: práctica de la bilirrubina, $\mathrm{p}=0,158$; $\mathrm{B}$ : práctica de la creatinina, $\mathrm{p}=0,334$; C: práctica de la urea, $\mathrm{p}=0,428$.

\subsection{Valoración de la metodología ABP}

Como medida de la calidad de la aplicación de la metodología ABP, los 20 estudiantes de la muestra poblacional completaron una encuesta de satisfacción. El cuestionario constó de 4 preguntas validadas (16) acerca del desarrollo del trabajo colaborativo entre los alumnos durante las prácticas. La primera cuestión hizo referencia a la adecuación de la información y orientaciones proporcionadas por el docente para solucionar los problemas mediante el trabajo en grupo de manera autónoma, que fue contestada afirmativamente por el $90 \%$ de los alumnos participantes. La segunda cuestión hizo referencia a la dinámica de grupo de las prácticas, contestando un afirmativamente el $65 \%$ del alumnado al ítem que indicaba que el grupo trabajó colectivamente, y un $30 \%$ al ítem que indicaba que el trabajo se repartió entre los componentes del grupo, poniendo en común la información y los criterios al comienzo y al final de la práctica. En la tercera cuestión se hizo referencia a si los contenidos básicos para abordar las prácticas eran los adecuados, respondiendo afirmativamente el $95 \%$ de los alumnos. La última pregunta del cuestionario evaluó el origen de las diferentes fuentes de información que utilizó el alumnado en el desarrollo de las prácticas con metodología ABP. En este caso, las respuestas fueron diversas, siendo un $70 \%$ las que hacían referencia a que la información había sido obtenida de diversas fuentes.

Las siguientes dos cuestiones del formulario consistieron en preguntas validadas (18), que hicieron referencia a la comparación de la metodología $\mathrm{ABP}$ con la tradicional. Las respuestas a estas dos cuestiones mostraron un porcentaje igualado al 50\% respecto a qué metodología (tradicional o ABP) encontraron más sencilla los alumnos (Figura 5). Asimismo, en la cuestión en la que se hizo referencia a qué metodología pensaban que aprendían más, el $70 \%$ del alumnado contestó con la metodología ABP (Figura 5B). 
A. Experimentalmente, ¿qué tipo de metodología encuentras más sencilla?

A

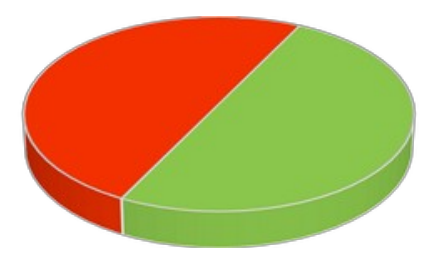

B. ¿Con qué metodología piensas que aprendes más?
Figura 5. Resultados de la pregunta 5 (A) y 6 (B) del cuestionario sobre la metodología aplicada.

En cuanto a la de la valoración de la práctica docente, destaca el alto grado de satisfacción general entre los estudiantes. El 80\% del alumnado consideró muy adecuada la labor del docente. El 95\% de los alumnos estuvo de acuerdo con que el profesor dominaba los contenidos, y el 100\% lo estuvo con que el docente facilitaba la comprensión de los contenidos básicos necesarios para realizar sus prácticas de manera adecuada. Asimismo, el 95\% contestó de manera afirmativa a las cuestiones que hicieron referencia a la utilidad de las pistas proporcionadas por el profesor. Por último, el 95\% del alumnado estuvo de acuerdo con que el profesor motivó al alumnado en la realización de las prácticas con metodología ABP.

\section{Discusión}

En las últimas dos décadas, con la progresiva implantación en España de las propuestas innovadoras del EEES, por las que se promueve el aprendizaje a lo largo de la vida y el "aprender a aprender", se ha hecho evidente la necesidad de innovar en el aula (3). Para ello, se aplican metodologías que se centren en el alumno, y que se sabe dan buenos resultados en el proceso de enseñanza-aprendizaje como es el "Aprendizaje Basado en Problemas" (10). Con éste, se pretende que mediante el trabajo colaborativo, el alumnado alcance un aprendizaje significativo. No obstante, aun habiéndose probado su eficacia en estudiantes universitarios de carreras prácticas, como las sanitarias de Enfermería y Medicina $(13,16,19,20,21)$, no se ha aplicado en la FP. Por ello, se hace necesario el desarrollo de metodologías como el ABP en este nivel educativo, así como la realización de estudios que evalúen el impacto de la aplicación de esta metodología innovadora en la Formación profesional. Es por esto, que nuestro trabajo es pionero, ya que se aplica a un nivel de enseñanza en el que prácticamente no se ha llevado a cabo investigación educativa de los beneficios que pueden conllevar la aplicación de la metodología ABP, y, además, compara los resultados obtenidos en actividades realizadas con esta metodología, con los resultados obtenidos a partir de la aplicación de la metodología tradicional, mostrando que la metodología ABP produce una mejoría de estos. 


\subsection{La metodología del Aprendizaje Basado en Problemas mejora los} resultados de los alumnos

Nosotros hemos observado que cuando se aplica metodología ABP en la realización de las prácticas de laboratorio de un Ciclo Formativo, las notas medias de los alumnos muestran una mejoría, comparadas con las obtenidas tras aplicar una metodología tradicional. Los resultados de nuestro estudio concuerdan, en líneas generales, con los obtenidos en otros estudios realizados en Enseñanza Superior, al encontrarse una mejoría en las notas de los alumnos tras aplicar metodología $\operatorname{ABP}(17,22)$.

El análisis cualitativo de las notas medias obtenidas por los alumnos en la práctica de la determinación del ácido úrico cuando se aplicó la metodología ABP mostró que esta diferencia estadísticamente significativa, no siendo así en los resultados de las otras tres prácticas, esto es, la determinación de la creatinina, la bilirrubina y la urea. Este resultado puede deberse a diferentes factores, de los cuales uno de los que más influyen es el tamaño de la muestra, ya que el número de alumnos participantes en el estudio fue de 20, es decir, muy bajo para obtener resultados estadísticamente significativos. Esto difiere de los dos estudios anteriormente mencionados, en los que el número de estudiantes presentes fue de 76 y 68 , respectivamente $(17,22)$.

Otro factor limitante que podría estar influyendo de manera importante en el nivel de significación de los resultados, es el tiempo de incorporación o aplicación de la nueva metodología en el aula. Este factor puede justificar que, en la mayoría de las prácticas, las diferencias encontradas en los resultados tras aplicar ambas metodologías no hayan sido estadísticamente significativas. Este proceso es gradual, no se lleva a cabo de manera eficaz en cuestión de un día, ya que es algo nuevo para el alumnado y necesita un periodo de adaptación $(17,22)$. Este último no ha tenido lugar en nuestro estudio, ya que sólo se ha desarrollado en dos sesiones, por tanto, se asemeja más a una fase de familiarización que de adaptación.

Por último, la duración y la novedad de las técnicas aplicadas en las prácticas pueden constituir un factor influyente en la significancia de los resultados. Aunque todas las prácticas tienen una dificultad similar al pertenecer al mismo bloque temático y determinarse mediante la utilización del espectrofotómetro, unas son más largas que otras. De esta manera, a los estudiantes puede resultarles más difícil realizar una práctica más extensa de lo habitual, o bien aplicando una metodología novedosa para ellos.

4.2. Los alumnos valoran de manera positiva la aplicación de la metodología Aprendizaje Basada en Problemas en la realización de las prácticas de laboratorio

En la valoración de la metodología ABP por parte de los estudiantes, el 95\% estuvo de acuerdo con que el grado de conocimientos básicos para abordar las prácticas fue suficiente, y el $90 \%$ lo estuvo con que la ayuda proporcionada por el docente fue adecuada para desarrollar la práctica con autonomía. Nuestros resultados presentan muchas similitudes con los recogidos por otros autores, un 97\% y un 92\%, en estos ítems, respectivamente, que aplicaron la metodología ABP en niveles de enseñanza semejantes a la FP de Grado superior (17). Estos resultados refuerzan la idea 
que el profesor puede ejercer un papel de apoyo para facilitar un aprendizaje significativo en los alumnos, transfiriendo el papel protagonista del proceso de enseñanza-aprendizaje a los alumnos (7).

Asimismo, la aplicación de la metodología ABP permite que el alumnado amplíe la búsqueda de información a distintas fuentes y sea capaz de seleccionar qué información es relevante para llegar a solucionar el problema en cuestión (13). Este hecho está apoyado por nuestros resultados, en los que el $70 \%$ de los estudiantes consultó diversas fuentes de información antes de llegar a la solución del problema. La consulta y el contraste de diversas fuentes de información son ventajas del método que ayudan a alcanzar un pensamiento crítico que favorece el análisis de la información y, por tanto, el autoaprendizaje $(11,23)$.

\subsection{El trabajo en grupo mejora el aprendizaje significativo y colaborativo} en la realización de prácticas de laboratorio de un ciclo formativo

La distribución de los alumnos en pequeños grupos de trabajo (3-4 personas) favorece el trabajo colaborativo y centra la carga del aprendizaje en el alumnado (24). Hubo una tendencia general de los alumnos a realizar las prácticas de manera grupal (65\%), hecho muy importante en nuestro estudio, ya que, de acuerdo con otros autores, el alumnado valora positivamente el trabajo en grupo; se incentivan las habilidades comunicativas (escuchar, cooperar, tener en cuenta las ideas diferentes y resolver conflictos) y se aprende sobre la importancia del trabajo en equipo para solucionar un problema a través de la búsqueda, selección e integración de diferentes temas, construyendo de esta manera el conocimiento, a la vez que se desarrollan habilidades y aptitudes sociales (25).

Frente a este resultado, un porcentaje reducido de los alumnos reconoció haber repartido el trabajo entre los componentes del grupo, pero poniendo en común las conclusiones obtenidas e intercambiando información durante todo el proceso. Para evitar este tipo de comportamiento que reproduce un aprendizaje tradicional e individualista, sería preciso realizar un seguimiento más exhaustivo del alumnado en el futuro con el fin de asegurarse que trabajan colectivamente durante todo el proceso de las prácticas. De esta forma, podría reducirse este porcentaje, que es común con los obtenidos en estudios similares (17), para mejorar las condiciones necesarias para el desarrollo de un aprendizaje colaborativo al aplicar la metodología ABP.

4.4. El alumnado considera a la metodología $A B P$ más útil para su aprendizaje, pero no demuestra preferencia hacia ella frente a la tradicional

Al comparar la sencillez de la metodología tradicional con la metodología $\mathrm{ABP}$, más compleja y que requiere la implicación del alumnado, no se observa una especial preferencia de los alumnados por la metodología ABP respecto a la tradicional. Una explicación plausible a este hecho es que la metodología $\mathrm{ABP}$ requiere un mayor esfuerzo y tiempo (26), ya que buscar información para entender las prácticas y no seguir simplemente un protocolo, como si fuera una receta para la realización de estas, puede resultarles una tarea ardua y provocar cierto rechazo en ellos. Además, los estudiantes podrían sufrir un rechazo inicial al cambio, debido a que esta metodología es muy diferente a la que están acostumbrados a realizar (25). Sin embargo, y a pesar de que no hay diferencias en las preferencias entre ambas 
metodologías, un porcentaje elevado de los alumnos (70\%) consideró aprender más con la metodología $\mathrm{ABP}$, concordando con los resultados extraídos de estudios realizados con estudiantes de Medicina, que tras la aplicación de la metodología ABP, percibieron estar mejor preparados para resolver problemas en su vida laboral (27). Por último, un tercer factor a considerar en esta disparidad de resultados es el poco tiempo de aplicación de la metodología ABP en nuestro estudio. Esta metodología es nueva para los alumnos, que, como ya hemos apuntado, necesitan un tiempo de adaptación y un tiempo más prolongado de aplicación, de al menos uno o dos cursos académicos (16). Por tanto, un factor fundamental para tener en cuenta en este tipo de metodología es el tiempo en la que se aplica, ya que el proceso de aprendizaje es largo y el estudiantado tiene que adaptarse a los nuevos métodos de enseñanza.

\subsection{La metodología ABP motiva a los alumnos para mejorar su proceso de enseñanza-aprendizaje}

La motivación es uno de los elementos más importantes en el proceso de enseñanza-aprendizaje, siendo esencial para el correcto aprendizaje del alumnado (28). Para que el alumnado encuentre motivación, es necesario, en primer lugar, que las actividades o problemas que se les plantea sean de una dificultad acorde a los conocimientos de los estudiantes, es decir, que puedan abordarlos con éxito. Además, la actividad debe ser atractiva para ellos, viendo en ella un reto interesante que deben conseguir resolver notoriamente y que no les provoque aburrimiento o ansiedad (29). Se hace evidente que, en este proceso, el papel del profesor es muy importante. Éste es el encargado de diseñar actividades dentro del currículo y llevar a cabo metodologías innovadoras con el fin de que los estudiantes se encuentren motivados y alcancen un aprendizaje significativo (28). Se hace evidente que la motivación es uno de los ítems más importantes en los cuestionarios de autoevaluación y satisfacción del alumnado. En el presente trabajo, los alumnos valoraron la capacidad del profesor para motivarlos en la realización de una nueva metodología que no habían llevado a cabo con anterioridad, manifestando un porcentaje elevado de ellos (el 95\%) que el docente los motivó para resolver los supuestos prácticos mediante la metodología ABP. Este hecho se vio reforzado con el feedback entre docente y alumnos que se encontraron cómodos y satisfechos con la metodología ABP, concordando con los resultados obtenidos en otros estudios en los cuales la metodología ABP favoreció la motivación del alumnado, desarrolló las habilidades necesarias para el aprendizaje significativo y reforzó así el sentimiento de profesionalidad en el futuro ámbito laboral del alumnado $(13,29,30)$.

Por último, otra característica de la metodología ABP que favorece la motivación es el conllevar un proceso de búsqueda de información planteando retos y favoreciendo el aprendizaje autodirigido, lo que genera un sentimiento de diversión y orgullo tanto en el alumnado como en el docente, mejorando de esta manera la motivación y el proceso de aprendizaje (26). Este sentimiento de diversión y motivación pudo observarse durante el desarrollo de los supuestos prácticos, tanto en el alumnado como en el profesor.

\subsection{Propuestas de futuro}

Nuestra propuesta de futuro consiste principalmente es aplicar este estudio en una población más grande, con un número muestral mayor. Esto nos permitirá, por un lado, trabajar con una mayor cantidad de contenidos 
para ver si la metodología $\mathrm{ABP}$ mejora el aprendizaje de los alumnos de contenidos académicos diversos, ya que el actual estudio se ha centrado únicamente en una Unidad de Trabajo, lo que ha influido en los resultados obtenidos. Sin embargo, debido a la actual situación pandémica, que comenzó cuando se estaba realizando nuestro estudio, no nos es posible realizarlo con la misma población. Para ver la eficacia de esta metodología, la aplicaremos en estudiantes del primer curso del ciclo formativo (que consta de dos cursos), y terminaremos el estudio en el segundo curso. Con ello implementaremos la metodología $\mathrm{ABP}$ durante un tiempo más prolongado, para que, como ya hemos sugerido, en un mínimo de dos cursos académicos se observan resultados significativos (16). Por último, hay que tener en cuenta para futuros estudios el hecho de que se han realizado pocos estudios de aplicación de metodologías no tradicionales en la Formación Profesional. A la vista de lo anterior, sería interesante seguir investigando la eficacia de la implementación de metodologías innovadoras para determinar cómo afectan los diferentes métodos de enseñanza en el aprendizaje del alumnado en una etapa educativa de carácter predominantemente profesionalizante, como es el caso de la Formación Profesional.

\section{Conclusiones}

- En este estudio observamos una tendencia de los resultados hacia una mejoría con la metodología ABP con respecto a la metodología tradicional, a pesar de que no hemos obtenido diferencias estadísticamente significativas entre ambas metodologías en tres de los cuatro cuestionarios relacionados con las prácticas desarrolladas, lo que indica que la aplicación de metodología ABP puede ser útil en las aulas de prácticas de Ciclos Formativos para mejorar los resultados de enseñanza-aprendizaje, y, como consecuencia, los resultados académicos de los alumnos.

- El Aprendizaje Basado en Problemas es una metodología que tiende a promover el aprendizaje colaborativo cuando los estudiantes resuelven los problemas propuestos en grupos de trabajo.

- La opinión generalizada en el alumnado, independientemente del tipo de alumno, edad o conocimientos previos, apunta a que con la metodología $\mathrm{ABP}$, la información proporcionada y el modo de actuación del docente, que ejerce un papel pasivo, son adecuados para alcanzar un aprendizaje significativo.

- La metodología del Aprendizaje Basado en Problemas parece ser útil para que los estudiantes de Ciclos Formativos de la especialidad Biosanitaria logren un aprendizaje significativo de los contenidos, actitudes y destrezas que se pretende que alcancen en las prácticas de laboratorio.

Financiación: Este estudio no ha sido financiado.

Agradecimientos: Agradecemos su colaboración para la realización de este trabajo al Instituto de Enseñanza Secundaria Miguel de Cervantes de Murcia, a los estudiantes que tan altruistamente participaron en el estudio y, en particular, al docente Cesáreo Cerón González, tutor de las prácticas del Máster de Formación del Profesorado de la Universidad de Murcia, ya que sin su colaboración y dedicación no habría sido posible.

Declaración de conflicto de interés: Los autores declaran no tener ningún conflicto de intereses. 


\section{Anexo I. Cuestionario de evaluación de la metodología ABP}

1.- Durante la resolución de las prácticas se ha procurado atender a los grupos de modo que su trabajo tuviera un elevado grado de autonomía. ¿Cómo crees que se ha desarrollado realmente el trabajo?

a) Ha sido como una receta. No se ha favorecido la autonomía e iniciativa del grupo.

b) Ha habido cierta autonomía e iniciativa, pero se ha proporcionado excesiva información y se han resuelto dudas y situaciones que hubiera superado el grupo.

c) La información y orientaciones proporcionadas han sido las adecuadas para desarrollar nuestro trabajo con un elevado grado de autonomía.

2.- Las prácticas se plantearon para ser desarrolladas en equipo. ¿Cuál de las siguientes afirmaciones refleja mejor el método de trabajo seguido en el grupo?

a) El trabajo ha sido repartido y realizado individualmente, con muy poca colaboración.

b) El trabajo ha sido repartido y realizado individualmente, pero al menos, al principio y al final, hay un encuentro para unificar criterios, intercambiar información...

c) El grupo siempre ha trabajado colectivamente.

3-El grado de conocimientos básicos para abordar las prácticas lo considero...

a) Insuficiente. No he entendido términos y conceptos básicos.

b) El nivel ha sido bajo respecto a los contenidos del curso.

c) Suficiente. El tema desarrollado está adaptado al nivel del curso.

4.- La información utilizada ha sido obtenida de...

a) Internet, principalmente.

b) Manuales y libros de texto, principalmente.

c) Diversas fuentes: internet, libros, revistas, apuntes, etc.

d) Otras fuentes.

5.- Experimentalmente, ¿qué tipo de metodología encuentras más sencilla?

a) Tradicional.

b) Aprendizaje basado en problemas.

6.- ¿Con qué metodología piensas que aprendes más?

a) Tradicional.

b) Aprendizaje basado en problemas.

\section{Referencias}

1. Comisión para la Renovación de las Metodologías Educativas en la Universidad. Propuesta para la renovación de las metodologías educativas en la Universidad. Ministerio de Educación y Ciencia. Consejo de Organización Universitaria. Edita: Secretaría General Técnica. 2006.

2. Frenk J, Chen L, Bhutta Z A, Cohen J, Crispf N, Evans T, Finebergh H, Garcia P J, Ke Y, Kelley P, Kistnasamy B, Meleis A, Naylor D, Pablos-Méndez A, Reddyn S, Scrimshaw S, Sepúlveda J, Serwadda D, Zurayk H. Profesionales de la salud para el nuevo siglo: transformando la educación para fortalecer los sistemas de salud en un mundo interdependiente. Rev Peru Med Exp Salud Publica, 2011, 28, 337-341. 
3. Maquilón J. La formación del profesorado en el siglo XXI: propuestas ante los cambios económicos, sociales y culturales, 1a ed.; Editum: Universidad de Murcia, Murcia, España, $2011,22,1-343$.

4. Leiva C. Conductismo, cognitivismo y aprendizaje. Tec Marcha, 2005, 18, 66-73.

5. Moreno G, Martínez R, Moreno M, Fernández M, Guadalupe S. Acercamiento a las Teorías del Aprendizaje en la Educación Superior. Revista digital UNIANDES Episteme, 2017, 4, 48-60.

6. Ausubel D P. Teoría del aprendizaje significativo. Psicología Educativa. Un punto de vista cognoscitivo 1999, 3, 1 - 10.

7. Ballester A, Gayoso P, Payeras J, Vicens G. El aprendizaje significativo en la práctica y didáctica de la geografía: prácticas del Seminario de aprendizaje significativo. Educación y Pedagogía, 2002, 14, 97-110.

8. Ausubel D P, Novak J D, Hanesian H. Significado y aprendizaje significativo. Psicología Educativa. Un punto de vista cognoscitivo, $2^{\text {a }}$ ed.; México: Trillas, 1993, 47-55.

9. Rodríguez M. Monogràfic sobre Aprenentatge significatiu. Revista Electrónica de Investigación e Innovación Educativa y Socioeducativa, 2011, 3, 29-50.

10. Barrows H S. El aprendizaje basado en problemas en la medicina y más allá: una breve descripción. New Directions for Teaching and Learning; Wilkerson L \& Gijselaers W H, Eds. Editorial: Jossey-Bass Publishers, San Francisco, E.E.U.U., 1986, 68, 3-11. DOI: https://doi.org/10.3102/00346543075001027.

11. Solaz-Portolés J, Sanjosé-López V, Gómez-López A. Aprendizaje basado en problemas en la Educación Superior: una metodología necesaria en la formación del profesorado. Didáctica de las Ciencias Experimentales y Sociales, 2011, 25, 177-186. http://hdl.handle.net/10550/21337.

12. Gijbels D, Dochy F, Van den Bossche P, Segers M. Effects of problem-based learning: A meta-analysis. Rev Educ Res, 2005, 75, 27-61. DOI: https://doi.org/10.3102/00346543075001027.

13. Egido I, Aranda $R$, Cerrillo $R$, De la Herrán A, De Miguel S, Gómez M, Hernández R, Izuzquiza D, Murillo F J, Pérez M. Estrategia metodológica y organizativa del currículum para la calidad de la enseñanza en los estudios de Magisterio. Aprendizaje basado en problemas (ABP). Revista Interuniversitaria de Formación del Profesorado, 2006, 20, 137149. https://www.redalyc.org/articulo.oa?id=27411311007.

14. Morales-Bueno $P$, Landa-Fitzgerald V. Aprendiaje Basado en Problemas. Problem-Based Learning. Theoria, 2004, 13, 145-157.

15. Hmelo-Silver C E. Problem-based learning: What and how do students learn? Educ Psych Rev, 2004, 16, 235-266. DOI: https://doi.org/10.1023/B:EDPR.0000034022.16470.f3.

16. Kelly O, Finlayson O. A hurdle too high? Students' experience of a PBL laboratory module. Chem. Educ. Res. Pract., 2009, 10, 42-52. DOI: https://doi.org/10.1039/B901459B.

17. Llorens-Molina J A. El aprendizaje basado en problemas como estrategia para el cambio metodológico en los trabajos de laboratorio. Quim. Nova, 2010, 33, 994-999. DOI: http://dx.doi.org/10.1590/S0100-40422010000400043.

18. González, C. Aplicación del “Aprendizaje Basado en Problemas” en los estudios de Grado en Enfermería. Tesis Doctoral. Universidad de Valladolid. 2012.

19. Norman, G. R. Problem-solving skills, solving problems and problem-based learning. Med. Educ., 1988, 22, 279-286. https://doi.org/10.1111/j.1365-2923.1988.tb00754.x

20. Barrows, H. S. Problem-Based, Self-directed Learning. JAMA, 1983, 250, 3077-3080. https://doi.org/10.1001/jama.1983.03340220045031. 
21. Coles, C. R. Differences between conventional and problem-based curricula in their students' approaches to studying. Med. Educ., 1985, 19, 308-309. https://doi.org/10.1111/j.1365-2923.1985.tb01327.x.

22. Saiz C, Fernández S. Pensamiento crítico y aprendizaje basado en problemas cotidianos. REDU, 2012, 10, 325-346. http://www.pensamiento-critico.com/archivos/bolonia.pdf.

23. Molina J A, García A, Pedraz A, Marcos, Antón M A. Aprendizaje basado en problemas: una alternativa al método tradicional. Revista de la Red Estatal de Docencia Universitaria, 2007, 3, 79-85.

24. Lillo F G. Aprendizaje Colaborativo en la Formación Universitaria de Pregrado. Rev. Psic. Univ. Viña del Mar, 2013, 2, 109-142.

25. Fernández M, J-N García, Caso A, Arias O. El aprendizaje basado en problemas: revisión de estudios empíricos internacionales. Rev. Educ., 2006, 341, 397-418.

26. Wood D F. Problem based learning. BMJ, 2003, 326, 328-330. DOI: https://doi.org/10.1136/bmj.326.7384.328.

27. Jones A, McArdle P J, O'Neill P A. Perceptions of how well graduates are prepared for the role of pre-registration house of(r)cer: A comparison of outcomes from a traditional and an integrated PBL curriculum. Med. Educ., 2002, 36, 16-25. DOI: 10.1046 / j.13652923.2002.01105.x.

28. Ospina J. La motivación, motor del aprendizaje. Rev. cienc. salud (Bogotá) 2006, 4, 158160.

29. Núñez J C. (2009). Motivación, aprendizaje y rendimiento académico. Actas do X Congresso Internacional Galego-Português de Psicopedagogia. Braga: Universidade do Minho, 2009, 41-67.

30. Bligh J, Lloyd-Jones G, Smith G. Early effects of a new problem-based clinically oriented curriculum on students' perceptions of teaching. Med. Educ., 2000, 34, 487-489. DOI: 10.1046/j.1365-2923.2000.00447.x.

(C) 2020 por los autores. Enviado para su publicación en acceso abierto bajo los términos y condiciones de la licencia Creative Commons Attribution (CC BY) (http://creativecommons.org/licenses/by/4.0/). 